\title{
STABILIZATION OF LINEAR BOUNDARY CONTROL SYSTEMS OF PARABOLIC TYPE: AN ALGEBRAIC APPROACH
}

\author{
Takao Nambu \\ Department of Applied Mathematics \\ Kobe University \\ Nada, Kobe 657-8501, Japan \\ nambu@godel.seg.kobe-u.ac.jp
}

\section{INTRODUCTION}

We consider in this paper the stabilization problem of a class of linear boundary control systems of parabolic type by means of feedback control. Our boundary control system with state $u=u(t, \cdot)$ is described by the differential equation:

$$
\frac{\partial u}{\partial t}+\mathcal{L} u=0, \quad \tau u=\sum_{k=1}^{M} f_{k}(t) \tilde{h}_{k}(\xi), \quad u(0, x)=u_{0}(x) .
$$

Here, $f_{k}(t)$ denote control inputs; $\tilde{h}_{k}(\xi)$ actuators on the boundary; and $(\mathcal{L}, \tau)$ a system of linear differential operators in a bounded domain $\Omega$ of $\mathbb{R}^{m}$ with the boundary $\Gamma$ consisting of a finite number of smooth components of $(m-1)$ dimension. Actually, let $\mathcal{L}$ denote a uniformly elliptic differential operator of order 2 in $\Omega$ defined by

$$
\mathcal{L} u=-\sum_{i, j=1}^{m} \frac{\partial}{\partial x_{i}}\left(a_{i j}(x) \frac{\partial u}{\partial x_{j}}\right)+c(x) u
$$

where $a_{i j}(x)=a_{j i}(x)$ for $1 \leqslant i, j \leqslant m, x \in \bar{\Omega}$. Associated with $\mathcal{L}$ is the boundary operator $\tau$ defined by

$$
\tau u=\alpha(\xi) u(\xi)+(1-\alpha(\xi)) \frac{\partial u(\xi)}{\partial \nu}=\alpha(\xi) u(\xi)+(1-\alpha(\xi)) \sum_{i, j=1}^{m} a_{i j}(\xi) \nu_{i}(\xi) \frac{\partial u}{\partial x_{j}}
$$


where $\alpha(\xi)$ denotes a smooth function on $\Gamma$, satisfying $0 \leqslant \alpha(\xi) \leqslant 1$, and $\left(\nu_{1}(\xi), \ldots, \nu_{m}(\xi)\right)$ the unit outer normal at $\xi \in \Gamma$. The inner products in $L^{2}(\Omega)$ and $L^{2}(\Gamma)$ are denoted by $\langle\cdot, \cdot\rangle_{\Omega}$ and $\langle\cdot, \cdot\rangle_{\Gamma}$, respectively.

The output of the system (1.1) is given by

$$
\left\langle u, w_{k}\right\rangle_{\Omega}, \quad 1 \leqslant k \leqslant N, \quad w_{k} \in L^{2}(\Omega) .
$$

By feeding the output back into $f_{k}(t)$ 's, our task is to choose suitable feedback parameters so that the state $u(t, \cdot)$ decays exponentially as $t \rightarrow \infty$ for every initial state $u_{0}$. Stabilization results for (1.1) in the literature are limited to the case where $\alpha(\xi) \equiv 1$ (the Dirichlet boundary) or the case where $0 \leqslant \alpha(\xi)<1$ (the generalized Neumann boundary), e.g., $[1,3,7]$ : The stabilization has been achieved, mainly due to the fact that the structure of the fractional powers of the associated elliptic operator $L$ is entirely known $[2,5,8]$. In the generalized Neumann case, for example, set $x(t)=L_{c}^{-1 / 4-\epsilon} u(t, \cdot), 0<\epsilon<1 / 4$ for a large $c>0$, where $L_{c}=L+c$. Then $x(t)$ satisfies the differential equation with the homogeneous boundary condition [7]:

$$
\frac{d x}{d t}+L x=\sum_{k=1}^{M} f_{k}(t) L_{c}^{3 / 4-\epsilon} g_{k}, \quad x(0)=L_{c}^{-1 / 4-\epsilon} u_{0},
$$

where $g_{k} \in H^{2}(\Omega)$ denote the unique solutions to the boundary value problems: $(\mathcal{L}+c) g_{k}=0$ in $\Omega, \tau g_{k}=\tilde{h}_{k}$ on $\Gamma, 1 \leqslant k \leqslant M$ [4]. Thus the control enters the equation as a distributed input in this transformed equation. This has made the problem considerably easy. The above transform works just like an integral transform which makes the state $u$ smoother in space variables.

Our boundary condition is partly of the Dirichlet type on the set $\Gamma_{1}=$ $\{\xi \epsilon \Gamma ; \alpha(\xi)=1\} \neq \emptyset$ and partly of the generalized Neumann type on $\Gamma \backslash \Gamma_{1}$. Unfortunately the structure of $L_{c}^{\omega}$ with this boundary condition is not well known at present in the context of the fractional Sobolev spaces. Thus the above approach seems no more available in our problem. The objective of this paper is to develop an alternative approach to the stabilization. By introducing an algebraic transform $T$, the whole stabilization procedure is of an algebraic nature as the title shows.

A compensator is a differential equation in $\mathbb{R}^{\ell}$ written by

$$
\frac{d v}{d t}+B_{1} v=\sum_{k=1}^{N}\left\langle u, w_{k}\right\rangle_{\Omega} \xi_{k}+\sum_{k=1}^{N}\left\langle v, \zeta_{k}\right\rangle_{\mathbb{R}^{\ell}} \eta_{k}, \quad v(0)=v_{0} .
$$

Here, the matrix $B_{1}$; the vectors $\xi_{k}, \zeta_{k}, \eta_{k} ; \rho_{k}$ appearing just below; and the dimension $\ell$ are the parameters to be determined. By setting $f_{k}(t)=$ $\left\langle v, \rho_{k}\right\rangle_{\mathbb{R}^{\ell}}, 1 \leqslant k \leqslant N$, equations (1.1) and (1.3) become a closed loop system.

\section{PRELIMINARY RESULTS}

Let us begin with reviewing the well known spectral properties of $(\mathcal{L}, \tau)$. Set

$$
\hat{L} u=\mathcal{L} u, \quad \mathcal{D}(\hat{L})=\left\{u \epsilon C^{2}(\Omega) \cap C^{1}(\bar{\Omega}) ; \mathcal{L} u \epsilon L^{2}(\Omega), \tau u=0\right\} .
$$


The closure of $\hat{L}$ in $L^{2}(\Omega)$, denoted by $L$, is self adjoint and has a compact resolvent $(\lambda-L)^{-1}$. Thus there is a set of eigenpairs $\left\{\lambda_{i}, \varphi_{i j}\right\}$ such that [4]

(i) $\sigma(L)=\left\{\lambda_{1}, \lambda_{2}, \ldots\right\}, \quad-\infty<\lambda_{1}<\lambda_{2}<\cdots<\lambda_{i}<\cdots \rightarrow \infty$;

(ii) $\left(\lambda_{i}-L\right) \varphi_{i j}=0, \quad i \geqslant 1,1 \leqslant j \leqslant m_{i}(<\infty)$; and

(iii) the set $\left\{\varphi_{i j}\right\}$ forms a complete orthonormal system for $L^{2}(\Omega)$.

For $\lambda$ in $\rho(L)$ and $h$ in $C^{2+\omega}(\Gamma)$, the boundary value problem

$$
(\lambda-\mathcal{L}) u=0 \quad \text { in } \Omega, \quad \tau u=h \quad \text { on } \Gamma
$$

admits a unique solution $u \epsilon C^{2}(\Omega) \cap C^{1}(\bar{\Omega})$ such that $\mathcal{L} u$ is in $L^{2}(\Omega)$. The solution $u$ is denoted by $N_{\lambda} h$. Set $C=-\sum_{k=1}^{M}\left\langle\cdot, \hat{\rho}_{k}\right\rangle_{\Omega} N_{-c} h_{k}$, where $\hat{\rho}_{k} \in L^{2}(\Omega)$ are to be given later in (3.15). If $\lambda$ is in $\rho(L+C) \cap \rho(L)$, the problem

$$
(\lambda-\mathcal{L}-C) u=0 \quad \text { in } \Omega, \quad \tau u=h \text { on } \Gamma
$$

also admits a unique solution $u \epsilon C^{2}(\Omega) \cap C^{1}(\bar{\Omega})$ such that $\mathcal{L} u$ is in $L^{2}(\Omega)$. The solution is denoted by $N(\lambda) h$.

\section{MAIN RESULT}

Let us consider the feedback control system

$$
\begin{aligned}
& \frac{\partial u}{\partial t}+\mathcal{L} u=0, \quad \tau u=\sum_{k=1}^{M}\left\langle v, \tilde{\rho}_{k}\right\rangle_{\mathbb{R}^{\ell}} \tilde{h}_{k}(\xi), \quad u(0, x)=u_{0}(x) \epsilon L^{2}(\Omega) \\
& \frac{d v}{d t}+B_{1} v=\sum_{k=1}^{N}\left\langle u, w_{k}\right\rangle_{\Omega} \tilde{\xi}_{k}+\sum_{k=1}^{N}\left\langle v, \tilde{\zeta}_{k}\right\rangle_{\mathbb{R}^{\ell}} \tilde{\eta}_{k}, \quad v(0)=v_{0} \in \mathbb{R}^{\ell}
\end{aligned}
$$

Associated with (3.1) is an auxiliary feedback control system described by

$$
\begin{aligned}
& \frac{\partial u}{\partial t}+\mathcal{L} u=0, \quad \tau u=\sum_{k=1}^{M}\left\langle v, \rho_{k}\right\rangle_{\Omega} \tilde{h}_{k}(\xi), \quad u(0, x)=u_{0}(x) \epsilon L^{2}(\Omega) \\
& \frac{d v}{d t}+B v=\sum_{k=1}^{N}\left\langle u, w_{k}\right\rangle_{\Omega} \xi_{k}+\sum_{k=1}^{N}\left\langle v, \zeta_{k}\right\rangle_{\Omega} \eta_{k}, \quad v(0)=v_{0} \epsilon L^{2}(\Omega)
\end{aligned}
$$

Here, the differential equation for $v$ is the one in $L^{2}(\Omega)$, and

$$
B=L-\sum_{k=1}^{M}\left\langle\cdot, L_{c} \rho_{k}\right\rangle_{\Omega} N_{-c} h_{k}, \quad \mathcal{D}(B)=\mathcal{D}(L)
$$

where $L_{c}=L+c, c>-\lambda_{1} ; h_{k} \in C^{2+\omega}(\Gamma) ;$ and $\rho_{k} \in \mathcal{D}(\hat{L}), 1 \leqslant k \leqslant M$. Set

$$
\Phi_{c}=\left(\left\langle N_{-c} h_{k}, \rho_{j}\right\rangle_{\Omega} ; \begin{array}{c}
k \rightarrow 1, \ldots, M \\
j \downarrow 1, \ldots, M
\end{array}\right) .
$$


Here, the vectors $\rho_{j}$ 's will be determined by the stabilization problem for $B$. We may assume with no loss of generality that $\left(1-\Phi_{c}\right)^{-1}$ exists. The actuators $\tilde{h}_{k}$ 's in (3.1) are then defined by

$$
\left[\tilde{h}_{1} \ldots \tilde{h}_{M}\right]=\left[h_{1} \ldots h_{M}\right]\left(1-\Phi_{c}\right)^{-1} .
$$

Define the matrices $H_{i}$ of size $m_{i} \times M$ and $W_{i}$ of size $m_{i} \times N$ by

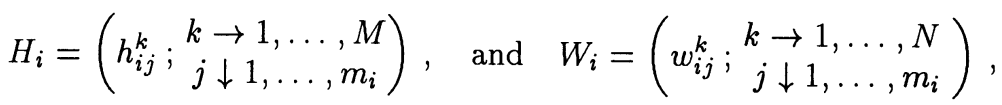

respectively, where

$$
h_{i j}^{k}=\left\langle h_{k}, \varphi_{i j}-\frac{\partial \varphi_{i j}}{\partial \nu}\right\rangle_{\Gamma}, \quad \text { and } \quad w_{i j}^{k}=\left\langle w_{k}, \varphi_{i j}\right\rangle_{\Omega} .
$$

Our main result is stated as follows:

Theorem 3.1. Suppose that $\lambda_{1}<\cdots<\lambda_{I} \leqslant 0<\lambda_{I+1}$, and that

$$
\operatorname{rank} H_{i}=\operatorname{rank} W_{i}=m_{i}, \quad 1 \leqslant i \leqslant I .
$$

Then, for any $0<\lambda<\lambda_{I+1}$, there exist parameters $\rho_{k}$ 's, $\xi_{k}$ 's, $\zeta_{k}$ 's and $\eta_{k}$ 's such that every solution to (3.2) with $u_{0}, v_{0} \epsilon L^{2}(\Omega)$ satisfies the estimate

$$
\|u(t, \cdot)\|+\|v(t, \cdot)\| \leqslant \text { const } e^{-\lambda t}\left\{\left\|u_{0}\right\|+\left\|v_{0}\right\|\right\}, \quad t \geqslant 0 .
$$

Eqn. (3.2) is reduced to (3.1) with some integer $\ell$, which admits a unique genuine solution $(u(t, x), v(t))$ for every $\left(u_{0}, v_{0}\right) \in L^{2}(\Omega) \times \mathbb{R}^{\ell}$ such that $\mathcal{L} u$ is bounded in $\left(t_{1}, t_{2}\right) \times \Omega, 0<{ }^{\forall} t_{1}<{ }^{\forall} t_{2}$. Every solution to (3.1) satisfies the estimate

$$
\|u(t, \cdot)\|+|v(t)|_{\ell} \leqslant \text { const } e^{-\lambda t}\left\{\left\|u_{0}\right\|+\left|v_{0}\right|_{\ell}\right\}, \quad t \geqslant 0
$$

Outline of the proof: First Step (Operator T). Let us consider the operator $B$ in (3.3) and determine $\rho_{k}$ 's in it. Assumption (3.7) implies that

$$
\operatorname{rank}\left(\left\langle N_{-c} h_{k}, \varphi_{i j}\right\rangle_{\Omega} ; \begin{array}{c}
k \rightarrow 1, \ldots, M \\
j \downarrow 1, \ldots, m_{i}
\end{array}\right)=\operatorname{rank} H_{i}=m_{i}, \quad 1 \leqslant i \leqslant I .
$$

Let $P_{I}$ denote the projection operator mapping $L^{2}(\Omega)$ onto $\operatorname{span}\left\{\varphi_{i j} ; 1 \leqslant i \leqslant\right.$ $\left.I, 1 \leqslant j \leqslant m_{i}\right\}$. According to the pole assignment theory of finite dimension [10], (see also [9]), there exist $\rho_{k}$ 's in $P_{I} L^{2}(\Omega)$ such that $\sigma(B)$ is contained in $\left\{\lambda \in \mathbb{C} ; \operatorname{Re} \lambda \geqslant \lambda_{I+1}\right\}$. The analytic semigroup $e^{-t B}$ thus satisfies the estimate

$$
\left\|e^{-t B}\right\| \leqslant \text { const } e^{-\lambda_{I+1} t}, \quad t \geqslant 0 .
$$

Let us introduce the algebraic transform $T$ in $\mathcal{L}\left(L^{2}(\Omega)\right)$ by

$$
p=T u=u-\sum_{k=1}^{M}\left\langle u, \rho_{k}\right\rangle_{\Omega} N_{-c} h_{k}
$$


The operator $T$ is injective, and $T^{-1} \epsilon \mathcal{L}\left(L^{2}(\Omega)\right)$ is expressed by

$$
u=T^{-1} p=p+\left[N_{-c} h_{1} \ldots N_{-c} h_{M}\right]\left(1-\Phi_{c}\right)^{-1}\langle p, \rho\rangle_{\Omega},
$$

where $\boldsymbol{\rho}$ denotes the transpose of $\left(\rho_{1}, \ldots, \rho_{M}\right)$. Set

$$
\zeta_{k}=\left(T^{-1}\right)^{*} w_{k}=\hat{w}_{k}, \quad \eta_{k}=-\xi_{k}, \quad 1 \leqslant k \leqslant N .
$$

Here, $\xi_{k}$ 's are to be determined in the Second Step. Then, $p-v$ satisfies

$$
\begin{aligned}
\frac{\partial(p-v)}{\partial t}+\mathcal{B}(p-v)= & \sum_{j, k=1}^{M}\left\langle p-v, \rho_{j}\right\rangle_{\Omega}\left\langle\tilde{h}_{j}, \rho_{k}-\frac{\partial \rho_{k}}{\partial \nu}\right\rangle_{\Gamma} N_{-c} h_{k} \\
& -\sum_{k=1}^{N}\left\langle p-v, \hat{w}_{k}\right\rangle_{\Omega} \xi_{k} \\
\tau(p-v)= & -\sum_{k=1}^{M}\left\langle p-v, \rho_{k}\right\rangle_{\Omega} \tilde{h}_{k}, \quad(p-v)(0, \cdot)=p_{0}-v_{0}
\end{aligned}
$$

where we have formally set $\mathcal{B}=\mathcal{L}-\sum_{k=1}^{M}\left\langle\cdot, L_{c} \rho_{k}\right\rangle_{\Omega} N_{-c} h_{k}$.

Second Step (Operator $K$ ). We introduce the operator $\hat{K}$ by

$$
\begin{gathered}
\hat{K} y=\mathcal{B} y-\sum_{j, k=1}^{M}\left\langle y, \rho_{j}\right\rangle_{\Omega}\left\langle\tilde{h}_{j}, \rho_{k}-\frac{\partial \rho_{k}}{\partial \nu}\right\rangle_{\Gamma} N_{-c} h_{k} \\
=\mathcal{L} y-\sum_{k=1}^{M}\left\langle y, \hat{\rho}_{k}\right\rangle_{\Omega} N_{-c} h_{k}, \quad \text { where } \\
\mathcal{D}(\hat{K})=\left\{y \epsilon C^{2}(\Omega) \cap C^{1}(\bar{\Omega}) ; \mathcal{L} y \in L^{2}(\Omega), \tau y=-\sum_{k=1}^{M}\left\langle y, \rho_{k}\right\rangle_{\Omega} \tilde{h}_{k} \text { on } \Gamma\right\} .
\end{gathered}
$$

Proposition 3.2. The operator $\hat{K}$ admits the closure $K$ which is densely defined in $L^{2}(\Omega)$. There exists a sector $\bar{\Sigma}_{-a}=\left\{\lambda-a \in \mathbb{C} ; \theta_{0} \leqslant|\arg \lambda| \leqslant\right.$ $\pi\}, 0<\theta_{0}<\pi / 2, a \in \mathbb{R}^{1}$, such that the resolvent of $K$ is expressed in $\bar{\Sigma}_{-a}$ as

$$
\begin{aligned}
(\lambda-K)^{-1}= & \left(\lambda-K^{0}\right)^{-1} \\
& -\left[N(\lambda) \tilde{h}_{1} \ldots N(\lambda) \tilde{h}_{M}\right](1+\Phi(\lambda))^{-1}\left\langle\left(\lambda-K^{0}\right)^{-1} \cdot \boldsymbol{\rho}\right\rangle_{\Omega},
\end{aligned}
$$

where $K^{0}=L-\sum_{k=1}^{M}\left\langle\cdot, \hat{\rho}_{k}\right\rangle_{\Omega} N_{-c} h_{k}, \mathcal{D}\left(K^{0}\right)=\mathcal{D}(L)$, and

$$
\Phi(\lambda)=\left(\left\langle N(\lambda) \tilde{h}_{k}, \rho_{j}\right\rangle_{\Omega} ; \begin{array}{c}
k \rightarrow 1, \ldots, M \\
j \downarrow 1, \ldots, M
\end{array}\right) .
$$

Proposition 3.3. The operator $T$ is an isomorphism which maps $\mathcal{D}(L)$ onto $\mathcal{D}(K)$. Furthermore, $K$ is similar to $L$, that is,

$$
T^{-1} K T=L, \quad T \mathcal{D}(L)=\mathcal{D}(K) .
$$


Associated with (3.14) is the differential equation for $y=p-v$ in $L^{2}(\Omega)$ :

$$
\frac{d y}{d t}+K y+\sum_{k=1}^{N}\left\langle y, \hat{w}_{k}\right\rangle_{\Omega} \xi_{k}=0, \quad y(0)=y_{0}=p_{0}-v_{0}
$$

Set $z=T^{-1} y$. Then, by Proposition 3.3, (3.17) is equivalent to

$$
\frac{d z}{d t}+L z+\sum_{k=1}^{N}\left\langle z, w_{k}\right\rangle_{\Omega} T^{-1} \xi_{k}=0, \quad z(0)=z_{0} .
$$

(Determination of $\xi_{k}$ 's) In view of the assumption (3.7) and the above equation for $z$, there exist $T^{-1} \xi_{k}$ 's in $P_{I} L^{2}(\Omega)$ and thus $\xi_{k}$ 's in $C^{2}(\Omega) \cap C^{1}(\bar{\Omega})$ such that the following estimate holds:

$$
\left\|\exp t\left(-K-\sum_{k=1}^{N}\left\langle\cdot, \hat{w}_{k}\right\rangle_{\Omega} \xi_{k}\right)\right\| \leqslant \text { const } e^{-\lambda_{I+1} t}, \quad t \geqslant 0 .
$$

Third Step (Equation for $p$ and $v$ ). The differential equation for $y$ and $v$ is the one in the product space $L^{2}(\Omega) \times L^{2}(\Omega)$ :

$$
\begin{gathered}
\frac{d}{d t}\left(\begin{array}{l}
y \\
v
\end{array}\right)+(F+G)\left(\begin{array}{l}
y \\
v
\end{array}\right)=\left(\begin{array}{l}
0 \\
0
\end{array}\right), \quad\left(\begin{array}{l}
y(0) \\
v(0)
\end{array}\right)=\left(\begin{array}{l}
y_{0} \\
v_{0}
\end{array}\right), \quad \text { where } \\
F\left(\begin{array}{l}
y \\
v
\end{array}\right)=\left(\begin{array}{ll}
K & 0 \\
0 & B
\end{array}\right)\left(\begin{array}{l}
y \\
v
\end{array}\right), \quad \text { and } \quad G\left(\begin{array}{l}
y \\
v
\end{array}\right)=\sum_{k=1}^{N}\left\langle y, \hat{w}_{k}\right\rangle_{\Omega}\left(\begin{array}{c}
\xi_{k} \\
-\xi_{k}
\end{array}\right) .
\end{gathered}
$$

The operator $-F-G$ generates an analytic semigroup. The equation for $p(=$ $y+v)$ and $v$ is written as, by setting $\Lambda=S(F+G) S^{-1}$ with $S=\left(\begin{array}{ll}1 & 1 \\ 0 & 1\end{array}\right)$,

$$
\frac{d}{d t}\left(\begin{array}{l}
p \\
v
\end{array}\right)+\Lambda\left(\begin{array}{l}
p \\
v
\end{array}\right)=\left(\begin{array}{l}
0 \\
0
\end{array}\right), \quad\left(\begin{array}{l}
p(0) \\
v(0)
\end{array}\right)=\left(\begin{array}{l}
p_{0} \\
v_{0}
\end{array}\right)
$$

Proposition 3.4. The operator $\Lambda$ is expressed by

$$
\Lambda\left(\begin{array}{l}
p \\
v
\end{array}\right)=\left(\begin{array}{c}
B\left(p+\sum_{k=1}^{M}\left\langle p-v, \rho_{k}\right\rangle_{\Omega} N_{-c} \tilde{h}_{k}\right)-\sum_{k=1}^{M}\left\langle p-v, \rho_{k}\right\rangle_{\Omega} \mathcal{B} N_{-c} \tilde{h}_{k} \\
-\sum_{j, k=1}^{M}\left\langle p-v, \rho_{j}\right\rangle_{\Omega}\left\langle\tilde{h}_{j}, \rho_{k}-\frac{\partial \rho_{k}}{\partial \nu}\right\rangle_{\Gamma} N_{-c} h_{k} \\
B v-\sum_{k=1}^{M}\left\langle p-v, \hat{w}_{k}\right\rangle_{\Omega} \xi_{k} \\
\mathcal{D}(\Lambda)=\left\{\left(\begin{array}{l}
p \\
v
\end{array}\right) ; p-v \in \mathcal{D}(K), \quad v \in \mathcal{D}(B)\right\} .
\end{array}\right.
$$


Fourth Step (Stabilization). The decay estimates (3.10) and (3.18) imply that, for any $\lambda^{\prime} ; \lambda<\lambda^{\prime}<\lambda_{I+1}$,

$$
\left\|e^{-t \Lambda}\right\|_{\mathcal{L}\left(L^{2}(\Omega) \times L^{2}(\Omega)\right)} \leqslant \text { const } e^{-\lambda^{\prime} t}, \quad t \geqslant 0 .
$$

This establishes the decay estimate (3.8), since $u=T^{-1} p$.

In order to obtain a finite-dimensional compensator, let us add a small perturbation $\Pi_{n}$ to (3.20) to obtain the equation:

$$
\begin{gathered}
\frac{d}{d t}\left(\begin{array}{l}
p \\
v
\end{array}\right)+\left(\Lambda+\Pi_{n}\right)\left(\begin{array}{l}
p \\
v
\end{array}\right)=\left(\begin{array}{l}
0 \\
0
\end{array}\right), \quad\left(\begin{array}{l}
p(0) \\
v(0)
\end{array}\right)=\left(\begin{array}{l}
p_{0} \\
v_{0}
\end{array}\right), \text { where } \\
0 \\
\Pi_{n}\left(\begin{array}{l}
p \\
v
\end{array}\right)=\left(\begin{array}{l}
\sum_{k=1}^{N}\left(\left\langle p-v, \hat{w}_{k}\right\rangle_{\Omega}\left(1-P_{n}\right) \xi_{k}-\left\langle v,\left(1-P_{n}\right) \hat{w}_{k}\right\rangle_{\Omega} P_{n} \xi_{k}\right) \\
+\sum_{k=1}^{M}\left\langle v, L_{c} \rho_{k}\right\rangle_{\Omega}\left(1-P_{n}\right) N_{-c} h_{k}
\end{array}\right)
\end{gathered}
$$

Since $\alpha_{n}=\left\|\Pi_{n}\right\|_{\mathcal{L}\left(L^{2}(\Omega) \times L^{2}(\Omega)\right)} \rightarrow 0$ as $n \rightarrow \infty$, we see that, for a large $n \geqslant I$,

$$
\left\|e^{-t\left(\Lambda+\Pi_{n}\right)}\right\|_{\mathcal{L}\left(L^{2}(\Omega) \times L^{2}(\Omega)\right)} \leqslant \text { const } e^{\left(-\lambda^{\prime}+\alpha_{n}\right) t} \leqslant \text { const } e^{-\lambda t}, \quad t \geqslant 0 .
$$

Set $v_{1}(t)=P_{n} v(t)$ for such an $n$. If $v_{0}$ belongs to $P_{n} L^{2}(\Omega)$, then $\left(p(t), v_{1}(t)\right)$ is a solution to (3.22). In other words, $v(t)$ remains in the finite-dimensional subspace $P_{n} L^{2}(\Omega)$ as long as the initial state $v_{0}$ belongs to $P_{n} L^{2}(\Omega)$.

Last Step (Regularity of $u$ and $v$ ). Henceforth we assume that $v_{0}$ belongs to $P_{n} L^{2}(\Omega)$ in (3.22) so that $v(t)$ remains in $P_{n} L^{2}(\Omega)$. It is shown -via a result in [6]- that the solution $p(t, x)$ in (3.22) is in $C^{2}(\Omega) \cap C^{1}(\bar{\Omega})$ for ${ }^{\forall} t>0$ and $\mathcal{L} p(t, x)$ is bounded in $\left(t_{1}, t_{2}\right) \times \Omega, 0<{ }^{\forall} t_{1}<{ }^{\forall} t_{2}$, and is a genuine solution to the initial-boundary value problem:

$$
\begin{aligned}
& \frac{\partial p}{\partial t}+\mathcal{B} p-\sum_{j, k=1}^{M}\left\langle p-v, \rho_{j}\right\rangle_{\Omega}\left\langle\tilde{h}_{j}, \rho_{k}-\frac{\partial \rho_{k}}{\partial \nu}\right\rangle_{\Gamma} N_{-c} h_{k}=0 \\
& \tau p=-\sum_{k=1}^{M}\left\langle p-v, \rho_{k}\right\rangle_{\Omega} \tilde{h}_{k}, \quad p(0, x)=p_{0}(x) .
\end{aligned}
$$

Let us derive the equation for $u=T^{-1} p$ and $v$. By applying Green's formula to a term of $\mathcal{B}$, the equation for $p$ is rewritten as

$$
\frac{\partial p}{\partial t}+\mathcal{L}_{c} p-\sum_{k=1}^{M}\left\langle\mathcal{L}_{c} p, \rho_{k}\right\rangle_{\Omega} N_{-c} h_{k}-c p=\frac{\partial p}{\partial t}+T \mathcal{L}_{c} p-c p=0 .
$$

Note that $\mathcal{L}_{c} p=\mathcal{L}_{c} u$ by (3.11). As for the boundary condition, we see that

$$
\tau u=\left[h_{1} \ldots h_{M}\right]\left(1-\Phi_{c}\right)^{-1}\langle p, \rho\rangle_{\Omega}-\left[\tilde{h}_{1} \ldots \tilde{h}_{M}\right]\langle p-v, \boldsymbol{\rho}\rangle_{\Omega}=\sum_{k=1}^{M}\left\langle v, \rho_{k}\right\rangle_{\Omega} \tilde{h}_{k} .
$$


Thus $(u, v)$ satisfies the differential equation

$$
\begin{aligned}
& \frac{\partial u}{\partial t}+\mathcal{L} u=0, \quad \tau u=\sum_{k=1}^{M}\left\langle v, \rho_{k}\right\rangle_{\Omega} \tilde{h}_{k}, \quad u(0, x)=u_{0} \epsilon L^{2}(\Omega) \\
& \frac{d v}{d t}+L v-\sum_{k=1}^{M}\left\langle v, L_{c} \rho_{k}\right\rangle_{\Omega} P_{n} N_{-c} h_{k} \\
& \quad-\sum_{k=1}^{N}\left\langle u, w_{k}\right\rangle_{\Omega} P_{n} \xi_{k}+\sum_{k=1}^{N}\left\langle v, P_{n} \hat{w}_{k}\right\rangle_{\Omega} P_{n} \xi_{k}=0, \quad v(0)=v_{0} \in P_{n} L^{2}(\Omega)
\end{aligned}
$$

Recall that the state of the compensator $v(t), t \geqslant 0$ in (3.25) remains in $P_{n} L^{2}(\Omega)$. Thus eqn. (3.25) is equivalent to (3.1) with $\ell=\operatorname{dim} P_{n} L^{2}(\Omega)=$ $m_{1}+\cdots+m_{n}$. The decay estimate (3.9) will be now clear. The uniqueness of solutions to (3.25) is also clear, since (3.25) is finally transformed to (3.22). This finishes the proof of Theorem 3.1. Q.E.D.

\section{References}

[1] R. F. Curtain, Finite dimensional compensators for parabolic distributed systems with unbounded control and observation, SIAM J. Control Optim., 22 (1984), 255-276.

[2] D. Fujiwara, Concrete characterization of the domain of fractional powers of some elliptic differential operators of the second order, Proc. Japan Acad. Ser. A Math Sci. 43 (1967), 82-86.

[3] J. S. Gibson and A. Adamian, Approximation theory for linear quadraticGaussian optimal control of flexible structures, SIAM J. Control Optim., 29 (1991), 1-37.

[4] D. Gilbarg and N. S. Trudinger, "Elliptic Partial Differential Equations of Second Order," 2nd ed., Springer-Verlag, New York, 1983.

[5] P. Grisvard, Caractérisation de quelques espaces d'interpolation, Arch. Rational Mech. Anal. 25 (1967), 40-63.

[6] S. Itô, "Diffusion Equations," Amer. Math. Soc., Providence, 1992.

[7] T. Nambu, On stabilization of partial differential equations of parabolic type: Boundary observation and feedback, Funkcial. Ekvac. 28 (1985), 267-298.

[8] T. Nambu, Characterization of the domain of fractional powers of a class of elliptic differential operators with feedback boundary conditions, J. Differential Equations 136 (1997), 294-324.

[9] Y. Sakawa, Feedback stabilization of linear diffusion systems, SIAM J. Control Optim., 21 (1983), 667-676.

[10] W. M. Wonham, On pole assignment in multi-input controllable linear systems, IEEE Trans. Automat. Control, AC-12 (1967), 660-665. 